\title{
Multidisciplinary management of primary signet-ring cell adenocarcinoma of the urinary bladder and the specific role of adjuvant radiotherapy
}

\author{
Krishna Reddy • James Moore • Benjamin Girdler • \\ Arlene Libby • Joshua Petit
}

Received: 18 February 2013 / Accepted: 1 April 2013 / Published online: 23 April 2013

(C) Springer-Verlag Berlin Heidelberg 2013

\section{Introduction}

Primary signet-ring cell (SRC) of the bladder is an aggressive, extremely uncommon subtype of bladder adenocarcinoma, comprising of $<1 \%$ of all primary bladder neoplasms [1]. A report based on analysis of the Surveillance, Epidemiology, and End Results (SEER) Program database found only 103 patients with SRC of the bladder diagnosed between 2001 and 2004, compared to 14,648 patients diagnosed with urothelial carcinoma [2]. The prognosis for patients with SRC is poor, attributed to presentation at advanced stages following asymptomatic progression [3], reported inefficacy of multimodality therapy [4], and possibly a more aggressive underlying biological phenotype [2] Diagnosis of bladder SRC can be challenging, both as it requires an extensive work-up to rule out more common metastatic adenocarcinomas [5], and comprehensive studies

K. Reddy $(\bowtie)$

Department of Radiation Oncology, University of Colorado

School of Medicine, 1665 Aurora Court, Suite 1032,

Aurora, CO 80045, USA

e-mail: Krishna.Reddy@ucdenver.edu

\section{J. Moore}

Department of Medical Oncology, Poudre Valley Health System, Fort Collins, CO, USA

B. Girdler

Department of Urologic Surgery, Poudre Valley Health System, Fort Collins, CO, USA
A. Libby
Department of Pathology, Poudre Valley Health System, Fort Collins, CO, USA
J. Petit
Department of Radiation Oncology, Poudre Valley Health System, Fort Collins, CO, USA

detailing the immunohistochemical profile of these tumors are understandably lacking [6]. Current treatment for this malignancy is often based on experiences gained from single institutional case series. Based on the aggressive natural history of bladder SRCs, treatment often features aggressive surgery in conjunction with chemo- and radiotherapy, although standardized recommendations for the use of adjuvant therapy are lacking. In this article, we present a case of locoregionally advanced SRC of the bladder, successfully managed with multimodality therapy. Literature regarding the diagnosis/work-up and management of bladder SRC is reviewed, with a focus on the specific role of adjuvant treatment.

\section{Case description}

This review was prompted by the case of a 60 -year-old woman who initially presented with urinary incontinence. A urologic work-up was undertaken, including cystoscopy, which demonstrated multiple submucosal lesions throughout the bladder. Biopsy of two of these lesions, involving the trigone and bladder dome, revealed histologic features consistent with adenocarcinoma of unknown primary origin. As the differential diagnosis included metastatic and endocervical adenocarcinomas, as well as a potential bladder primary lesion, a comprehensive diagnostic/staging work-up was undertaken. Studies performed included (1) PET/CT, which demonstrated a hypermetabolic mass in the superior-posterior bladder wall without evidence of regional nodal or metastatic disease; (2) gynecologic evaluation including Pap smear, which was negative; (3) pelvic MRI, which confirmed the PET/CT findings; (4) upper endoscopy and colonoscopy, which were negative; and (5) mammography, which was negative. Additional outside review of the biopsy pathology 
was also performed, with the consensus opinion that the bladder lesions were consistent with a primary adenocarcinoma of the bladder wall if clinical work-up did not reveal another primary malignancy. Following discussion in a multidisciplinary tumor conference, after careful consideration, aggressive locoregional therapy was recommended, given the absence of any known metastatic disease.

The patient underwent a radical cystectomy, hysterectomy, bilateral salpingo-oophorectomy, anterior vaginectomy, bilateral pelvic lymph node dissection, and creation of an Indiana pouch. Pathology revealed infiltrating poorly differentiated carcinoma with signet-ring cell features involving the bladder wall with extension into the anterior vaginal wall; positive surgical margins involving the vagina and parametrial soft tissue were observed. A representative pathologic section and summary of immunohistochemical findings is shown in Fig. 1. No positive lymph nodes were found among 13 excised. Six weeks following surgery, the patient underwent systemic chemotherapy with six cycles of cisplatin and gemcitabine (cisplatin $75 \mathrm{mg} / \mathrm{m}^{2}$, day 1 ; gemcitabine $1,000 \mathrm{mg} / \mathrm{m}^{2}$, days 1 and 8 ; every 21 days), which was well tolerated. Expected mild side effects included fatigue, nausea, and cytopenias. Longer-term persistent toxicities included peripheral sensory neuropathy and partial hearing loss. One unexpected finding was the development of bilateral inguinal lymphadenopathy; this was biopsy proven to be benign histiocytosis and did eventually selfresolve.

Following re-staging imaging studies which demonstrated no radiographic evidence of persistent/recurrent disease, she was treated with adjuvant radiotherapy, to further minimize the risk of locoregional recurrence. A seven-field intensitymodulated radiation therapy (IMRT) treatment plan was utilized to deliver a dose of $45 \mathrm{~Gy}$ in 25 fractions to the

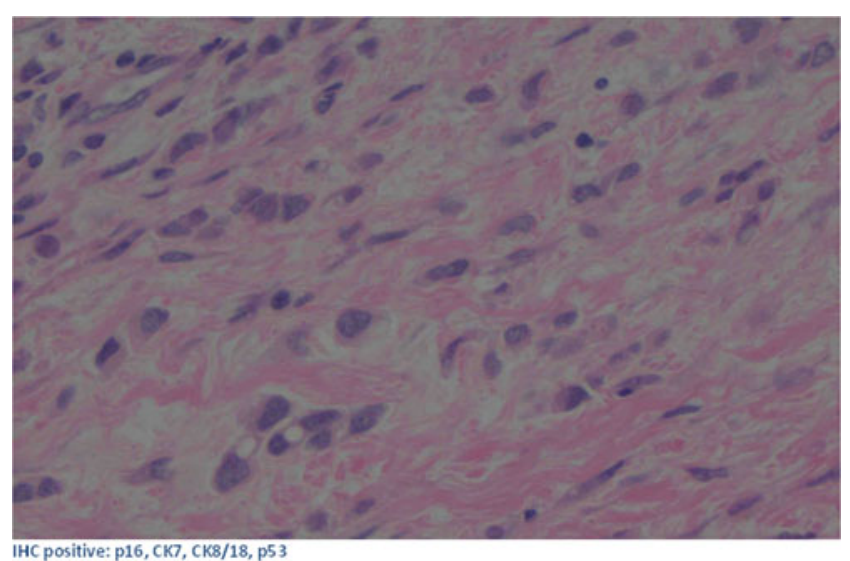

IHC negative: CK20, CK5\&6, CEA, CD45, mammoglobin, CDX-2, TTF-1, Mart-1, p63, WT-1, calretinin, ER, PR

Fig. 1 Tumor pathologic appearance and summary of immunohistochemical findings postoperative bed and regional lymph nodes (including pelvic and inguinal nodes) with daily fluoroscopic image guidance. Figure 2 depicts the patient's IMRT plan. Radiotherapy was tolerated well, with minimal treatment-related toxicities, specifically fatigue and local skin/mucosal irritation, which were easily managed with supportive care. The patient is now 2.5 years postcompletion of all therapy and doing very well, with no evidence of recurrent disease or late treatment-related toxicity, as well as an excellent self-reported, health-related quality of life. Informed consent from the patient was obtained prior to preparation of this case report. As the treatment described above was a multidisciplinary standard-of-care therapy, this case report was approved by the treating institution's IRB; no human (or animal) experimental/protocol study was conducted in our work.

\section{Discussion}

\section{Work-up/diagnosis}

SRCs are a variant of primary bladder adenocarcinomas, which collectively account for $0.5-2 \%$ of all malignant bladder neoplasms [5]. As in our patient discussed above, bladder adenocarcinomas are most often found in the trigone and posterior bladder wall $[5,7]$. Furthermore, the aggressive nature of SRCs leads to their frequent presentation with extravesical soft tissue extension $[5,7,8]$. Histologically, SRCs are poorly differentiated, round cell tumors with intracytoplasmic mucin without extracellular mucin, resembling a mammary lobular carcinoma except for large cell size [5]. The identification of individual cells displaying a signet-ring morphology is important for establishing a pathologic diagnosis [3, 9]; in fact, Thomas et al. [10] found significant correlations between the percentage of tumor containing signet-ring cells and both the presence of adverse disease features (unresectability of a primary tumor or regional lymph node metastasis) and overall survival.

Given the extremely low incidence and absence of any definitive histopathologic features, establishing the diagnosis of a primary bladder SRC requires clinical correlation and specific exclusion of another primary malignancy. A thorough clinical and radiographic evaluation is always warranted in cases of suspected primary bladder adenocarcinoma, to rule out bladder involvement from another primary malignancy, either via direct local extension or lymphatic/hematogenous metastasis. PET/CT, pelvic MRI, upper endoscopy/colonoscopy, as well as mammography/gynecologic examination should be performed, with specific consideration given to ruling out a primary neoplasm involving the GI tract (the colon, stomach, and appendix), prostate, genital tract, and breast. Imaging characteristics of a bladder adenocarcinoma have been reported, including increased bladder wall thickness and surrounding fat stranding [11]; overall, imaging features are more similar 
Fig. 2 IMRT plan. Dose distribution is shown overlaying representative axial, sagittal, and coronal sections. Orientation of the seven treatment fields is depicted (top right panel)
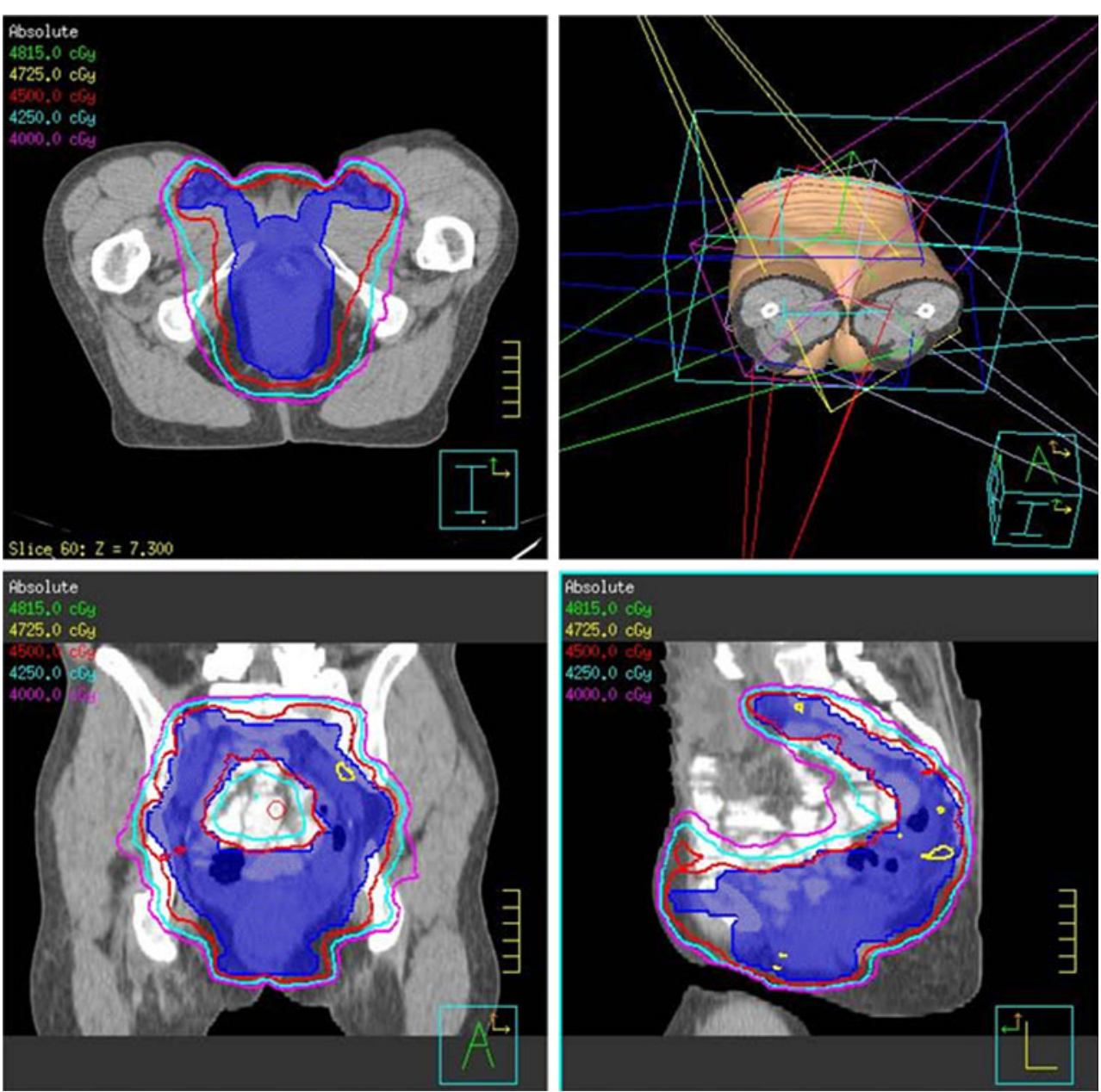

to those of adenocarcinomas involving other abdominal organs than to transitional cell bladder carcinomas.

From a histopathologic standpoint, immunohistochemical studies have sought to differentiate adenocarcinomas of a primary bladder origin from those involving the bladder as a result of local extension or metastasis from other primary malignancies. Lack of CDX2 and villin expression, for instance, has been found to be diagnostically valuable in distinguishing primary bladder adenocarcinomas from secondary colorectal carcinoma [12]. The utility of such primary bladder adenocarcinoma markers in identifying variants, such as SRC, however, has been questioned, as some studies of signet-ring cell carcinomas at other anatomic locations have demonstrated that their immunohistochemical profiles may differ from other primary adenocarcinomas occurring at these locations [10]. Still, Terada et al. [6] recently published a report analyzing the immunohistochemical profile of three cases of a primary SRC of the urinary bladder, with positive cytokeratin (CK) AE1/3, CK CAM5.2, CK8, CK18, CK19, CK20, p53, Ki-67, mucin (MUC) 1 and
MUC2, and negative CK34BE12, CK5/6, CK14, EMA, p63, vimentin, and MUC6 found.

\section{Multidisciplinary treatment and outcomes}

A SEER analysis by Wang et al. [2] found signet-ring carcinoma histology to be an independent negative predictor of survival, even after adjusting for grade, stage at diagnosis, patient characteristics, and treatment. Even among bladder adenocarcinomas, a group generally regarded as aggressive and associated with a poor prognosis, Zaghloul et al. [13] found that the SRC variant was associated with the worst 5-year disease-free survival (compared to mucinous, papillary, and unspecified subtypes). Given the rarity of SRC, diagnostic work-up and discussion of treatment options in a multidisciplinary setting, with input from urology, medical oncology, and radiation oncology, as well as pathology and diagnostic radiology, are always recommended. Generally, treatment involves surgery \pm adjuvant radiotherapy \pm chemotherapy. While the role of adjuvant RT and chemotherapy in SRC management has been questioned 
$[1,14]$, many agree that radical cystectomy is the most appropriate surgical option $[1,2]$.

\section{Chemotherapy}

No standard chemotherapy regimen exists for SRC of the bladder, and the use of various systemic agents with mixed success has been reported. Akamatsu et al. [1] reported the most commonly used chemotherapeutics utilized in the management of bladder SRC among Japanese institutions to be platinum-based drugs, followed by 5 -FU derivatives and anthracycline agents. Cobo-Dols et al. [15] utilized a combination of cisplatin and gemcitabine in a patient with pT4aN0M0 SRC of the bladder, with no evidence of disease noted at 8 months posttreatment. The successful use of intraarterial methotrexate and cisplatin with radiation was demonstrated by Ota et al. with posttreatment cystectomy revealing no evidence of carcinoma [16]. Furthermore, Hirano et al. [3] described the use of intra-arterial carboplatin in a patient with locally advanced, node-negative SRC of the bladder who declined surgery, with complete remission obtained. In principle, adjuvant chemotherapy may be considered for patients with adequate performance status and significant risk for distant failure.

Role of adjuvant RT in improving locoregional control in SRC

Adjuvant RT has been selectively utilized in the management of bladder SRC. Unfortunately, in most cases, the details regarding specific patient and/or disease factors which prompted the use of RT in these historical series are unknown; adjuvant RT was likely implemented in the presence of factors conferring a high risk of recurrence, such as positive margins or tumor infiltration into perivesical soft tissue. In their analysis of patients in the SEER database (2001-2004), Wang et al. [2] found that 0/14 patients with $\mathrm{SRC}$ of the bladder staged of $\angle \mathrm{T} 2$ received $\mathrm{RT}$, as compared to $13 / 65(20 \%)$ of patients staged of $\geq \mathrm{T} 2$. As postoperative radiotherapy is routinely utilized in the management of gastrointestinal, gynecologic, and urologic malignancies, including adenocarcinomas, to minimize the risk of pelvic recurrence, it would reasonable to expect a similar benefit for adjuvant RT post-cystectomy for adenocarcinoma of the bladder. To this point, among 142 patients with primary adenocarcinoma of the bladder, receiving radical cystectomy and pelvic lymphadenectomy, Zaghloul et al. [17] found that adjuvant RT improved 5-year disease-free survival from $38.2 \pm 6.1 \%$ in those receiving cystectomy alone to $56.7 \pm 7.5 \%(p=0.06)$. Five-year local control was $55.9 \pm 7.4 \%$ for surgery alone, as compared to $97 \pm 2.8 \%$ for post-op RT [17]. When limiting the comparison to patients with more locally advanced disease at presentation, specifically stages P2b, P3, and P4a, adjuvant RT improved 5-year DFS from $24.2 \pm 9.0 \%$ for cystectomy alone to $56.7 \pm 7.5 \%$, which was statistically significant $(p=0.01$ ) [17]. The benefit of adjuvant RT in this series was realized despite the inclusion of a higher proportion of patients with advanced stages, grade 3 tumors, and pelvic lymph node involvement in the post-op RT group [17]. While only $7 \%$ of patients in this series were reported to have SRC, we would not expect this particular histologic variant to respond to RT in a markedly different fashion compared to other subtypes of bladder adenocarcinoma. In fact, given the higher incidence of locoregional recurrence associated with SRC, if anything, the benefit of adjuvant RT may be greater in this population. While acknowledging the limited implications of a single case, the ability of adjuvant RT to facilitate durable locoregional control in our patient with pT4a disease and multiple positive margins also supports the efficacy for radiation therapy in SRC. In analyzing a mixed pathology series containing patients with adenocarcinoma, Zaghloul et al. [18] previously demonstrated that the benefit of post-op RT for patients with P3a, P3b, or P4a bladder tumors in improving disease-free survival and local control extended to all tumor types, pathologic stages, and histological

Table 1 Guidelines for adjuvant RT for primary signet-ring cell adenocarcinoma of the bladder

Indications for treatment

Volume delineation

Dose/fractionation/techniques
Stage of $\geq \mathrm{T} 2 \mathrm{~b}$ tumors, positive surgical margins, or pathologically positive lymph nodes

Postoperative bed based on preoperative imaging (CT and MRI), postoperative imaging, pathologic findings (such as involvement of adjacent organs), surgical clips, and consultation with urologic surgeon

Pelvic lymph nodes (including hypogastric, obturator, and internal/external iliac and presacral nodes)

If extension into adjacent organs is found at the time of cystectomy, cover relevant draining lymphatics (e.g., inguinal lymph nodes for vaginal involvement)

45-50.4 Gy (standard fractionation) to the postoperative bed and regional lymphatics

Additional adaptive boost to 60-66 Gy may be considered following the primary RT course in the setting of gross residual disease, if possible while respecting normal tissue constraints

IMRT can be employed to minimize dose to adjacent normal tissues, such as the bowel and rectum, when clinically indicated 
grades. Patients received either 1.25 Gy fractions three times per day up to a total dose of 37.5 Gy in 12 days, standard fractionated RT to a dose of $50 \mathrm{~Gy}$ in 5 weeks, or no further treatment following radical surgery [18]. The 5-year local control rate was $50 \%$ for surgery alone, compared to 87 and $93 \%$, respectively, for those receiving multiple daily fractionation and conventional fractionation [18]. Even among patients with transitional cell carcinoma of the bladder, a less aggressive histology compared to SRC, pelvic recurrence following cystectomy has been shown to be a major site of failure, up to $30 \%$ in some series [19]. In light of these and similar findings, Murthy and Zaghloul [20] recently argued for a reconsideration of the use of adjuvant RT post-cystectomy in patients with bladder cancer, both to help decrease the risk of local recurrence as well as potentially improve overall outcomes.

In addition to minimizing the risk of local recurrence in the setting of a positive surgical margin or known perivesical tissue/adjacent organ involvement, adjuvant RT following cystectomy has the additional potential benefit of decreasing the risk of a nodal recurrence. The extent of lymphadenectomy performed at the time of cystectomy has a known impact on recurrence risk [21]. In a SEER analysis of patients undergoing radical cystectomy for bladder cancer between 1988 and 1996, those with evaluation of 0 to 3 lymph nodes were determined to be at a significantly higher risk of death from bladder cancer than those with $>3$ examined [22]. Unfortunately, among patients undergoing cystectomy in this analysis, $40.3 \%$ had no lymph nodes examined, while an additional $27.6 \%$ had six or fewer nodes evaluated [22]. As regional nodal involvement can precede distant metastatic spread, coverage of draining lymphatic regions should be included in the design of any postcystectomy RT plan.

Patient selection and target delineation for post-cystectomy RT

Following surgery for bladder SRC, candidates for adjuvant RT include those at a high risk of local recurrence, including patients with (1) stage of $\geq \mathrm{T} 2 \mathrm{~b}$ tumors, (2) positive surgical margins, or (3) pathologically positive lymph nodes. In all cases, the postoperative bed should be covered in the RT plan. Delineation of the postoperative bed should be guided by preoperative imaging (CT and MRI); postoperative imaging; pathologic findings (such as involvement of adjacent organs); intraoperative aids, such as clips, placed at the time of the procedure; as well as consultation with the operating urologic surgeon. Pelvic lymph nodes (including hypogastric, obturator, and internal/external iliac and presacral nodes) should be encompassed in the target volume. Additionally, if extension into adjacent organs is found at the time of cystectomy, it is recommended that the relevant draining lymphatics supplying these structures are also covered, if possible. For example, in our case discussed above, local extension of disease into the anterior vaginal wall prompted inclusion of the inguinal lymph nodes within the target volume. A dose of 45 $50.4 \mathrm{~Gy}$, utilizing standard fractionation, to the postoperative bed and regional lymphatics is recommended. In the setting of gross residual disease following surgery, planning of an additional adaptive boost, up to a total dose of 60-66 Gy, may be cautiously attempted following completion of the initial course, if additional RT can be safely delivered while respecting normal tissue constraints. In planning adjuvant radiotherapy for our patient, the use of a boost was specifically discussed in a GU multidisciplinary conference with all treating physicians present; a boost was felt to not be indicated here based on intraoperative findings and final pathology review. IMRT can be employed to minimize dose to adjacent normal tissues, such as the bowel and rectum, when clinically indicated. A prone setup (using a belly board) should be considered to reduce bowel dose, if this is tolerable, and a reproducible position can be achieved (which can be facilitated with IGRT).

\section{Conclusion}

A thorough clinical and radiographic diagnostic/staging evaluation is always warranted in the setting of suspected primary signet-ring cell adenocarcinoma of the urinary bladder, given the rare nature of this neoplasm and the critical need to rule out local or metastatic bladder involvement from another primary malignancy. Locoregionally confined disease can be successfully managed with a combination of surgery, systemic therapy, and adjuvant radiotherapy. RT can help improve locoregional control, reducing the risk of a pelvic recurrence, and facilitated durable local control in our patient with pT4a disease and positive margins. Guidelines for the use of adjuvant RT in the management of bladder SRC are presented in Table 1.

Conflict of interest Krishna Reddy, James Moore, Benjamin Girdler, Arlene Libby, and Joshua Petit have no conflicts of interest.

\section{References}

1. Akamatsu S, Takahashi A, Ito M, Ogura K (2010) Primary signetring cell carcinoma of the urinary bladder. Urology 75(3):615-618

2. Wang J, Wang FW, Kessinger A (2012) The impact of signet-ring cell carcinoma histology on bladder cancer outcome. World J Urol 30(6):777-783. doi:10.1007/s00345-011-0718-8

3. Hirano Y, Suzuki K, Fujita K, Furuse H, Fukuta K, Kitagawa M et al (2002) Primary signet ring cell carcinoma of the urinary bladder successfully treated with intra-arterial chemotherapy alone. Urology 59(4):601

4. Erdoğru T, Kiliçaslan I, Esen T, Ander H, Ziylan O, Uysal V (1995) Primary signet ring cell carcinoma of the urinary bladder. Review of the literature and report of two cases. Urol Int 55(1):34-37 
5. Roy S, Parwani AV (2011) Adenocarcinoma of the urinary bladder. Arch Pathol Lab Med 135(12):1601-1605

6. Terada T (2012) Primary pure signet-ring cell adenocarcinoma of the urinary bladder: a report of three cases with an immunohistochemical study. Med Oncol 29(4):2866-2869. doi:10.1007/s12032-011-0122-7

7. Epstein JI, Amin MB, Reuter VE (2010) Glandular lesions. In: Epstein JI, Amin MB, Reuter VE (eds) Bladder biopsy interpretation: biopsy interpretation series, 2nd edn. Lippincott Williams \& Wilkins, Philadelphia, pp 150-176

8. Poore TE, Egbert B, Jahnke R, Kraft JK (1981) Signet ring cell adenocarcinoma of the bladder: linitis plastica variant. Arch Pathol Lab Med 105(4):203-204

9. Grignon DJ, Ro JY, Ayala AG, Johnson DE (1991) Primary signet-ring cell carcinoma of the urinary bladder. Am J Clin Pathol 95(1):13-20

10. Thomas AA, Stephenson AJ, Campbell SC, Jones JS, Hansel DE (2009) Clinicopathologic features and utility of immunohistochemical markers in signet-ring cell adenocarcinoma of the bladder. Hum Pathol 40(1):108-116

11. Hughes MJ, Fisher C, Sohaib SA (2004) Imaging features of primary nonurachal adenocarcinoma of the bladder. AJR Am J Roentgenol 183(5):1397-1401

12. Suh N, Yang XJ, Tretiakova MS, Humphrey PA, Wang HL (2005) Value of CDX2, villin, and alpha-methylacyl coenzyme A racemase immunostains in the distinction between primary adenocarcinoma of the bladder and secondary colorectal adenocarcinoma. Mod Pathol 18(9):1217-1222

13. Zaghloul MS, Nouh A, Nazmy M, Ramzy S, Zaghloul AS, Sedira MA et al (2006) Long-term results of primary adenocarcinoma of the urinary bladder: a report on 192 patients. Urol Oncol 24(1):13-20
14. Holmäng S, Borghede G, Johansson SL (1997) Primary signet ring cell carcinoma of the bladder: a report on 10 cases. Scand J Urol Nephrol 31(2):145-148

15. Cobo-Dols M, Alcaide J, Gutiérrez V, Gil S, Alés I, Villar E et al (2006) Primary signet-ring cell adenocarcinoma of the urinary bladder: a case report. Oncología 29(4):177-180

16. Ota T, Shimazui T, Hinotsu S (1995) Primary signet ring cell carcinoma of the bladder effectively treated with intraarterial chemotherapy and radiation therapy: a case report. Nishinihon J Urol 57:1019-1023

17. Zaghloul MS, Aziz SA, Nouh A, Mohran TZ, El-Shazely S, Saber A (2003) Primary adenocarcinoma of the urinary bladder: risk factors and value of postoperative radiotherapy. J Egypt Nat Cancer Inst 15(3): 193-200

18. Zaghloul MS, Awwad HK, Akoush HH, Omar S, Soliman O, el Attar I (1992) Postoperative radiotherapy of carcinoma in bilharzial bladder: improved disease free survival through improving local control. Int $\mathrm{J}$ Radiat Oncol Biol Phys 23(3):511-517

19. Hassan JM, Cookson MS, Smith JA Jr, Chang SS (2006) Patterns of initial transitional cell recurrence in patients after cystectomy. $\mathrm{J}$ Urol 175(6):2054-2057

20. Murthy V, Zaghloul MS (2007) Adjuvant radiotherapy in bladder cancer: time to take a fresh look? Urol Oncol 25(4):353-354

21. Skinner EC, Stein JP, Skinner DG (2007) Surgical benchmarks for the treatment of invasive bladder cancer. Urol Oncol 25(1):66-71

22. Konety BR, Joslyn SA, O'Donnell MA (2003) Extent of pelvic lymphadenectomy and its impact on outcome in patients diagnosed with bladder cancer: analysis of data from the Surveillance, Epidemiology and End Results Program data base. J Urol 169(3):946950 